\title{
Gas Bubbles Investigation in Contaminated Water Using Optical Tomography Based on Independent Component Analysis Method
}

\author{
Mohd Taufiq Mohd Khairi, Sallehuddin Ibrahim, \\ Mohd Amri Md Yunus, and Mahdi Faramarzi \\ Department of Control and Mechatronics Engineering, Faculty of Electrical Engineering, \\ Universiti Teknologi Malaysia, 81310 Skudai, Johor, Malaysia \\ Correspondence should be addressed to Sallehuddin Ibrahim; salleh@fke.utm.my
}

Received 3 September 2015; Revised 18 March 2016; Accepted 13 April 2016

Academic Editor: Augusto Beléndez

Copyright (C) 2016 Mohd Taufiq Mohd Khairi et al. This is an open access article distributed under the Creative Commons Attribution License, which permits unrestricted use, distribution, and reproduction in any medium, provided the original work is properly cited.

\begin{abstract}
This paper presents the results of concentration profiles for gas bubble flow in a vertical pipeline containing contaminated water using an optical tomography system. The concentration profiles for the bubble flow quantities are investigated under five different flows conditions, a single bubble, double bubbles, $25 \%$ of air opening, $50 \%$ of air opening, and $100 \%$ of air opening flow rates where a valve is used to control the gas flow in the vertical pipeline. The system is aided by the independent component analysis (ICA) algorithm to reconstruct the concentration profiles of the liquid-gas flow. The behaviour of the gas bubbles was investigated in contaminated water in which the water sample was prepared by adding $25 \mathrm{~mL}$ of colour ingredients to 3 liters of pure water. The result shows that the application of ICA has enabled the system to detect the presence of gas bubbles in contaminated water. This information provides vital information on the flow inside the pipe and hence could be very significant in increasing the efficiency of the process industries.
\end{abstract}

\section{Introduction}

Information about the flow regime in a process vessel or pipelines is important so as to design an accurate, safe, and low cost conveying system in various applications such as chemical engineering and nuclear engineering [1]. The flow regime identification is also vital in evaluating the performance of a process system since incorrect analysis from the system can lead to the reduction of production rates. Hence, an appropriate instrumentation system is essential in providing vital information on the flow patterns. The tomography technique has been selected extensively in the industrial field as a tool to provide information about the phase and spatial distribution without interrupting the process flow [2]. Before being applied in the industry, the technique has been successfully implemented in the medical field where it is responsible for capturing images of body tissue and detecting tumour [3]. A tomography system consists of several parts such as sensors, signal conditioning circuit, a data acquisition system, and a computer. There are many types of sensors that have been used in process tomography, that is, electrical capacitance, electrical resistance, ultrasonic, and optical. In this paper, an optical sensing technique has been selected to be integrated into the tomography system due to its low cost, being straightforward, and having a better dynamic response [4]. Many researchers have published their result using the optical sensor in order to monitor the movement of gas, liquid, and solid. Idroas et al. [5] have successfully applied an optical tomography system for particle sizing identification using sample of beads and irregular shaped nut. Rzasa and Plaskowski [6] have presented gas bubbles measurement using an optical tomography system in a vertical aeration column. Ibrahim et al. [7] proposed a concentration measurement using halogen bulbs as light projectors to reconstruct images of small and large gas bubbles. This paper describes the analysis of gas bubble detection in a pipeline filled with 
contaminated water. Investigation on gas bubble behaviour in industry such as food and pharmaceutical is vital since the existence of unwanted bubbles can reduce the quality of product [8]. Previous research on gas bubbles measurement in the tomography field was conducted by Rzasa [9], Jin et al. [10], and Ayob et al. [11] which used pure water as a liquid. Generally, the unwanted gas bubbles are in the form of opaque liquid such as oil and paint. Therefore, this paper proposed a technique for imaging the gas bubbles in which the investigation was carried out using opaque liquid.

\section{Mathematical Modelling}

Mathematical modelling is an essential part in optical tomography. This section discusses the optical attenuation model and the application of independent component analysis. The first part will discuss the optical attenuation modelling for the presence of gas bubbles in contaminated water. The second part elaborated the application of ICA method to detect the distribution of gas bubbles.

2.1. Optical Attenuation Model. Light experienced attenuation when it traverses from one medium to another medium. This attenuating process is due to scattering and refraction of light when it travels through a different medium such as water and gas. This paper highlighted the absorption and attenuation model and neglected the light scattering effect since it is difficult to perform the modelling due to the random shape [12]. Beer-Lambert's Law is a popular law regarding the investigation of attenuation and absorption of the light energy. The law can be expressed as

$$
V_{\mathrm{R}}=V_{\mathrm{T}} \exp \left[-\alpha_{\mathrm{w}} l_{\mathrm{w}}-\alpha_{\mathrm{a}} l_{\mathrm{a}}\right]
$$

where $V_{\mathrm{R}}$ is the receiving sensor voltage, $V_{\mathrm{T}}$ is the voltage of the transmitter, $\alpha_{\mathrm{w}}$ is the attenuation coefficient of water medi$\mathrm{um}\left(\mathrm{mm}^{-1}\right), \alpha_{\mathrm{a}}$ is the attenuation coefficient of air medium $\left(\mathrm{mm}^{-1}\right), l_{\mathrm{w}}$ is the path length of water $(\mathrm{mm})$, and $l_{\mathrm{a}}$ is the path length of air $(\mathrm{mm})$. The reference attenuation coefficients for water and air are $\alpha_{\mathrm{w}}=0.0287 \mathrm{~mm}^{-1}$ and $\alpha_{\mathrm{a}}=0.0142 \mathrm{~mm}^{-1}$ [12]. The value of pipe diameter is $l_{\mathrm{w}}=100 \mathrm{~mm}$ and the length of small bubble is assumed to be $l_{\mathrm{a}}=5 \mathrm{~mm}$. The value of $V_{\mathrm{T}}$ is being set to 5 volts $(\mathrm{V})$ which was taken from the transmitter value programmed using the Peripheral Interface Controller (PIC) Microcontroller. Two cases are investigated: water with no gas bubble presence and water with gas bubble presence.

2.1.1. Water with No Gas Bubble Presence. The arrangement of the transmitter and receiver when there is no gas bubble is illustrated in Figure 1. Light from the transmitter traversed water without the interruption of bubble. The value of the attenuation coefficient for water is entered in (2) along with $100 \mathrm{~mm}$ of water path length. The value of the receiver voltage $V_{\mathrm{R}}$ is obtained by

$$
\begin{aligned}
& V_{\mathrm{R}}=5 \exp [-(0.0287)(100)-(0)], \\
& V_{\mathrm{R}}=0.2835 \mathrm{~V} .
\end{aligned}
$$

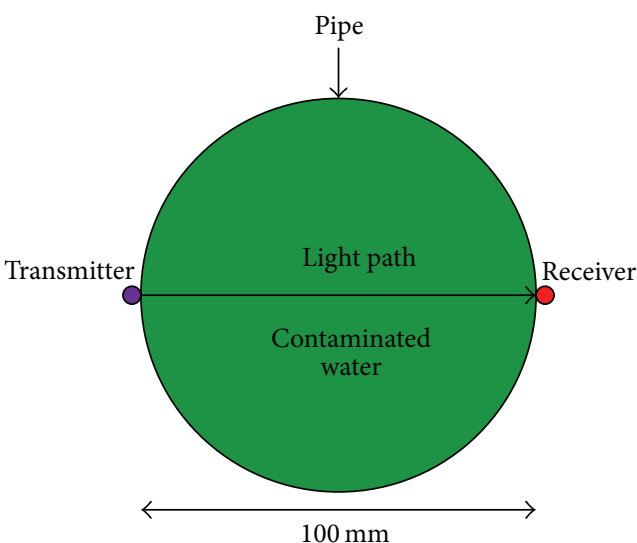

FIGURE 1: The light path in water when there is no gas bubble.

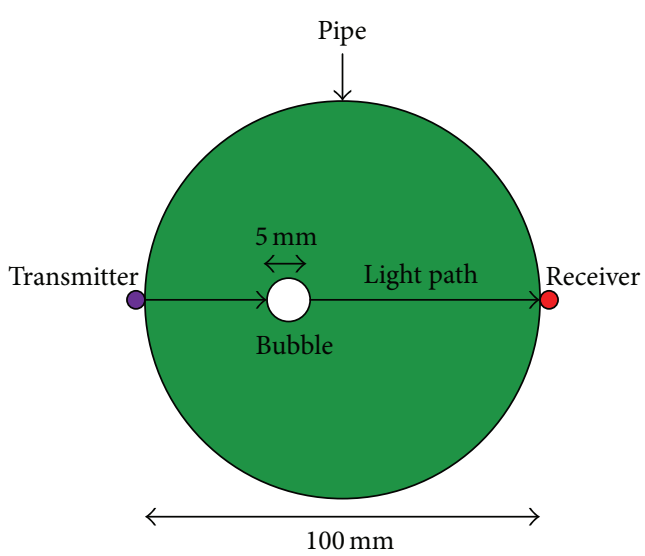

Figure 2: The condition of light path in water with gas bubble presence in contaminated water.

2.1.2. Water with Gas Bubble Presence. The aim of this modelling is to determine the change of parameters $V_{\mathrm{R}}$ and $\exp (-\alpha l)$ when a single gas bubble intercepts the transmitting light as shown in Figure 2. In this case, the illustration neglects the reflection and scattering effect when light beam passed through the bubble. The value of the receiving voltage $V_{\mathrm{R}}$ when light passed through bubble and water can be determined as follows:

$$
\begin{aligned}
& V_{\mathrm{R}}=5 \exp [-(0.0287)(100-5)-(0.0142)(5)], \\
& V_{\mathrm{R}}=0.3050 \mathrm{~V} .
\end{aligned}
$$

The values of $V_{\mathrm{R}}$ when there is no gas bubble and when gas bubble is present are compared. The existence of bubble in water resulted in a higher value of $V_{\mathrm{R}}$ as indicated in (5) compared to when no bubble is present as indicated in (3). Light experienced less attenuation when gas bubble is present. When there is a bubble, light needs to pass two dissimilar mediums: water and gas. Hence the exponential value is higher compared to when no bubble exists. The values of $V_{\mathrm{R}}$ and $\exp (-\alpha l)$ are predicted to increase when more and 


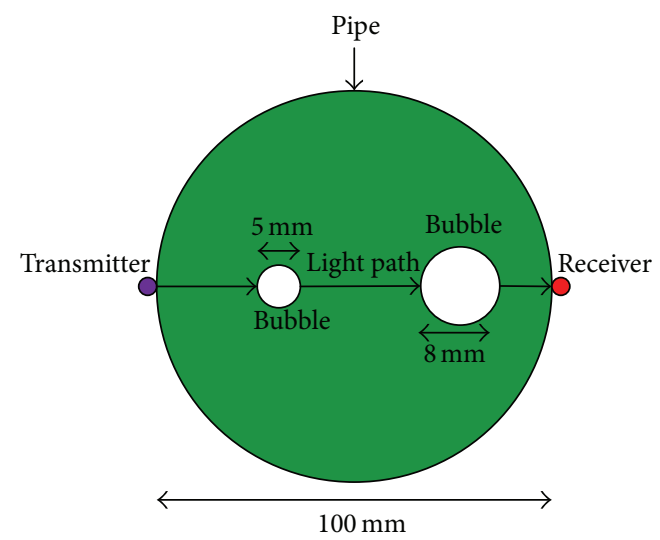

FIgURE 3: The light path when double model is present in contaminated water.

bigger size bubbles intercepted the light before it reaches the receiver's side. The illustration and an example of calculation of $V_{\mathrm{R}}$ for double bubble model can be seen in Figure 3. Related equations are given in

$$
\begin{aligned}
V_{\mathrm{R}} & =5 \exp [-(0.0287)(100-13)-(0.0142)(5) \\
& -(0.0142)(8)] \\
V_{\mathrm{R}} & =0.3425 \mathrm{~V} .
\end{aligned}
$$

2.2. Independent Component Analysis. Blind source separation (BSS) is a technique which separates a set of individual source signals from their mixtures without any specific knowledge as with very limited information about the source signals $[13,14]$. An adaptive system is required to solve this problem. Independent component analysis (ICA) is a method which is extensively used for extracting the mixture signals to individual signal [13]. The method is developed by Jutten and Herault at the end of 1980s for retrieving the original sound from the mixed sound signals in the microphone [15]. The method is different from the Principle Component Analysis (PCA) method where PCA is focused in finding the principle components which consist of linear combination of the observed variables, whereas ICA is concerned with discovering the independent components from the observed variables [16]. ICA has been applied in many areas such as telecommunications [17], image processing [18], gas-liquid phase separation in tomography [14], and biomedical applications [19]. A simple example on the application of ICA is shown in Figure 4. In Figure 4, the voice signals from two speakers are mixed together in each microphone. The crowded voices signals also add up and resulted in noise being produced. By using ICA, the original voice signal for both speakers can be acquired.

The general model of ICA is described as

$$
X=A S \text {, }
$$

where $X$ is $n \times m$ matrix of mixture of source signals, $A$ is $n \times d$ mixing matrix, and $S$ is $d \times m$ matrix denoting a source signal. The aim of ICA is to get the value of mixing matrix $(A)$ and/or source signal matrix $(S)$ given only realizations of the observation matrix of $X$. After the ICA process was executed, (10) converts to (11) where $W$ is the unmixing matrix and equals $A^{-1}$ :

$$
\widehat{S}=W X
$$

The illustration in Figure 5 can assist in understanding the concept of ICA technique. After going through the ICA process, the estimation of source signal $\widehat{S}$ is produced and it looks similar to the original signal, $S(t)$. Moreover, the ICA has formed the separating matrix which was produced alongside the output signal at the output side.

Among the most widely used ICA algorithms are FastICA and Infomax [20]. FastICA provides recovery of independent sources by employing the higher order statistics and the estimation process is done one by one [13]. The algorithm separates the source signals based on their non-Gaussianity and it finds one independent component at a time instead of solving the mixing matrix [20]. The FastICA algorithm is as follows [21]:

(1) Choose an initial weight vector, $w$.

(2) Let

$$
w^{+}=E\left\{x g\left(w^{\mathrm{T}} x\right)\right\}-E\left\{g^{\prime}\left(w^{\mathrm{T}} x\right)\right\} w
$$

where $w^{+}$is a temporary variable used to calculate weight vector, $E\{\cdots\}$ is averaging over all columnvectors of matrix $x, w^{\mathrm{T}}$ is a transpose of weight vector, $x$ is input data, and $g$ is the derivative of nonquadratic used in the contrast function for solving ICA problem.

(3) Let

$$
w=\frac{w^{+}}{\left\|w^{+}\right\|} .
$$

(4) If not converged, go back to (7).

The expression "converged" means the value is either old or new for $w$ in the same direction. The advantages of FastICA are that it is parallel and simple for computation process and requires small memory size [22]. For the Infomax algorithm, it is based on maximizing the information transferred in a network of nonlinear units and it is proposed by Bell and Sejnowski [23]. The algorithm is derived from a maximization principle between inputs and nonlinear outputs. The entropy can be described as

$$
H\left(s_{1}, s_{2}\right)=H\left(s_{1}\right)+H\left(s_{2}\right)-I\left(s_{1}, s_{2}\right) .
$$

$H\left(s_{1}, s_{2}\right)$ is the conditional entropy where the entropy of $s_{1}$ is conditional on $s_{2} . H\left(s_{1}\right)$ and $H\left(s_{2}\right)$ are the entropy of $s$ and $I\left(s_{1}, s_{2}\right)$ is mutual information. From this equation, maximizing the joint entropy of the outputs amount has led to minismising the mutual information [13]. The disadvantage of Infomax is that the function does not converge properly if step size is wrongly chosen [24]. 


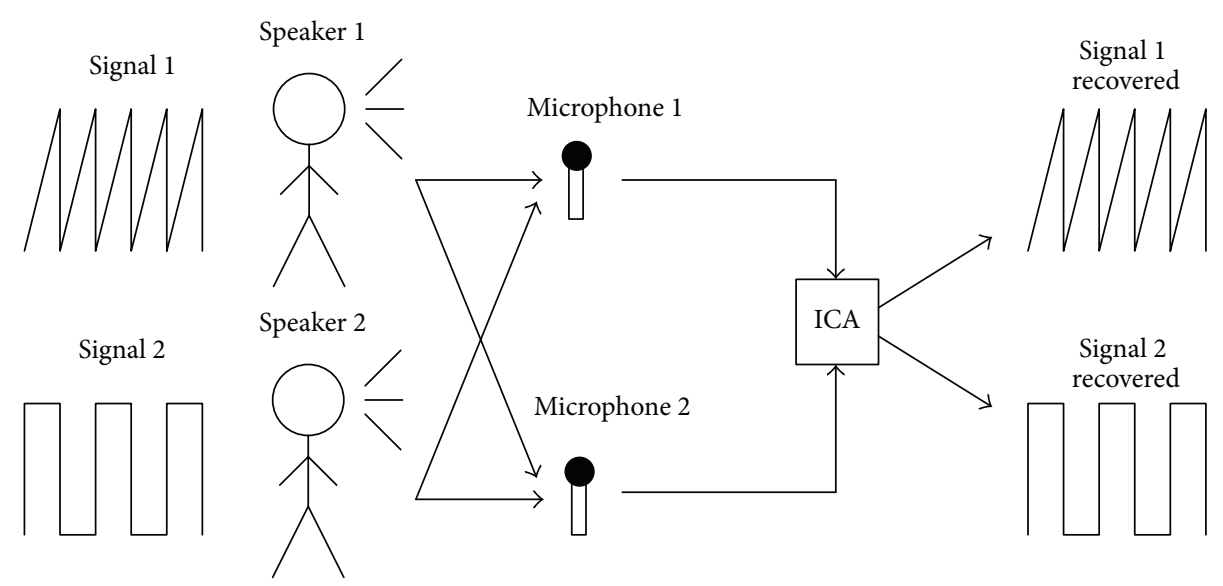

FIgURE 4: A simple example for understanding the ICA concept [13].

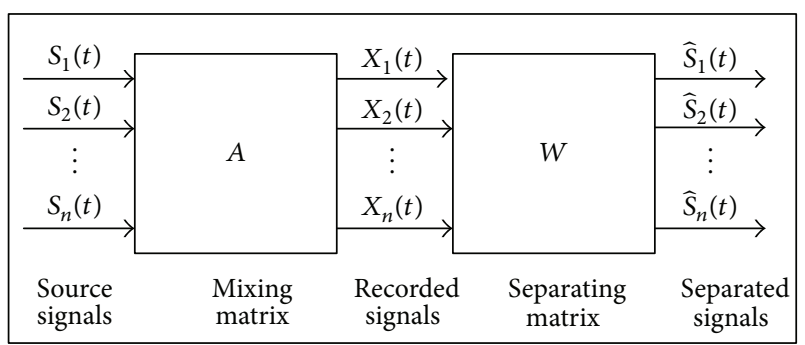

FIGURE 5: ICA block diagram.

This research emphasized the separation of the transmitters signals which were mixed in the receivers. In ICA, there is some ambiguity that should be handled properly. Firstly, the separated signals indicated that the amplitude of the signals is not identical as the source signals. Secondly, the separating matrix is formed in the arbitrary row [20]. A similar parameter from input and output that can be investigated in ICA is the frequency value. Therefore, each transmitter is set to have a different duty cycle in order to facilitate the rearrangement of the separating matrix row. An in-depth explanation about the rearrangement procedure is discussed in Section 4. For simplicity, the modelling for the algorithm is described using four transmitters and four receivers as shown in Figure 6 while the actual system consists of eighteen transmitters and eighteen receivers. This is because the explanation of the ICA concept using eighteen pairs of sensors is complex since the matrix equation is too long. Hence, in this section only four pairs of sensor and four absorption coefficients are chosen and are sufficient to show how ICA works. Transmitters 1 , 2,3 , and 4 are represented as TX1, TX2, TX3, and TX4, respectively, while receivers $1,2,3$, and 4 are represented as RX1, RX2, RX3, and RX4, respectively. The attenuation coefficients are represented as $\alpha_{1}, \alpha_{2}, \alpha_{3}$, and $\alpha_{4}$.

In this model, each receiver is supposed to detect light from all transmitters. Take, for example, receiver RX1. The four signals received at $\mathrm{RX1}$ have been mixed where each light

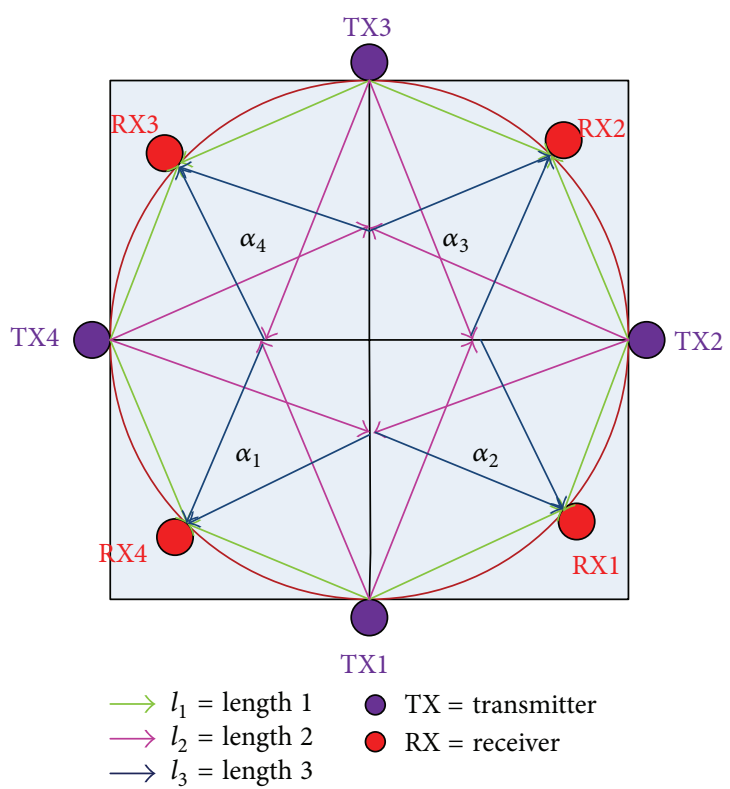

FIgURE 6: Model of four-sensor pair with four pixels.

has its own characteristics such as light length and absorption coefficient. The equation of receiver RX1 voltage $V_{\mathrm{R} 1}$ can be written as

$$
\begin{aligned}
V_{\mathrm{R} 1}= & V_{\mathrm{T} 1} \exp \left(-\alpha_{2} l_{1}\right)+V_{\mathrm{T} 2} \exp \left(-\alpha_{2} l_{1}\right) \\
& +V_{\mathrm{T} 3} \exp \left(-\alpha_{3} l_{2}\right)+V_{\mathrm{T} 3} \exp \left(-\alpha_{2} l_{3}\right) \\
& +V_{\mathrm{T} 4} \exp \left(-\alpha_{1} l_{2}\right)+V_{\mathrm{T} 4} \exp \left(-\alpha_{2} l_{3}\right),
\end{aligned}
$$

where $V_{\mathrm{T} 1}$ is transmitter TX1 voltage, $V_{\mathrm{T} 2}$ is transmitter TX2 voltage, $V_{\mathrm{T} 3}$ is transmitter TX3 voltage, and $V_{\mathrm{T} 4}$ is transmitter TX4 voltage, while $\alpha_{1}$ is attenuation coefficient for material 1 , $\alpha_{2}$ is attenuation coefficient for material 2, $\alpha_{3}$ is attenuation 
coefficient for material 3 , and $\alpha_{4}$ is attenuation coefficient for material 4 , whereas $l_{1}$ is length $1, l_{2}$ is length 2 , and $l_{3}$ is length 3 . Voltage transmitter $\left(V_{\mathrm{T}}\right)$ is a source signal when we analyse the light received by receiver. For example, as shown in Figure 6, receiver 1 (RX1) detects four lights from transmitter 1 (TX1), transmitter 2 (TX2), transmitter 3 (TX3), and transmitter 4 (TX4). The distribution of bubbles in a pipeline is estimated based on the parameters of $\exp (-\alpha l)$ and receiver output voltage $\left(V_{\mathrm{R}}\right)$ as indicated in (16). Equation (12) can be arranged as

$$
\begin{aligned}
V_{\mathrm{R} 1}= & V_{\mathrm{T} 1} \exp \left(-\alpha_{2} l_{1}\right)+V_{\mathrm{T} 2} \exp \left(-\alpha_{2} l_{1}\right) \\
& +V_{\mathrm{T} 3} \exp \left(-\alpha_{3} l_{2}-\alpha_{2} l_{3}\right) \\
& +V_{\mathrm{T} 4} \exp \left(-\alpha_{1} l_{2}-\alpha_{2} l_{3}\right) .
\end{aligned}
$$

$$
\begin{gathered}
X=c \\
{\left[\begin{array}{c}
V_{\mathrm{R} 1} \\
V_{\mathrm{R} 2} \\
V_{\mathrm{R} 3} \\
V_{\mathrm{R} 4}
\end{array}\right]=\left[\begin{array}{cccc}
e^{-\alpha_{2} l_{1}} & e^{-\alpha_{2} l_{1}} & e^{-\alpha_{3} l_{2}-\alpha_{2} l_{3}} & e^{-\alpha_{1} l_{2}-\alpha_{2} l_{3}} \\
e^{-\alpha_{2} l_{2}-\alpha_{3} l_{3}} & e^{-\alpha_{3} l_{1}} & e^{-\alpha_{3} l_{1}} & e^{-\alpha_{4} l_{2}-\alpha_{3} l_{3}} \\
e^{-\alpha_{1} l_{2}-\alpha_{4} l_{3}} & e^{-\alpha_{3} l_{2}-\alpha_{4} l_{3}} & e^{-\alpha_{4} l_{1}} & e^{-\alpha_{4} l_{1}} \\
e^{-\alpha_{1} l_{1}} & e^{-\alpha_{2} l_{2}-\alpha_{1} l_{3}} & e^{-\alpha_{4} l_{2}-\alpha_{1} l_{3}} & e^{-\alpha_{1} l_{1}}
\end{array}\right]\left[\begin{array}{c}
V_{\mathrm{T} 1} \\
V_{\mathrm{T} 2} \\
V_{\mathrm{T} 3} \\
V_{\mathrm{T} 4}
\end{array}\right] .}
\end{gathered}
$$
signified as a source signal $(S)$ : and receiver RX4 voltage are simplified as

Equations (13) to (14) are transformed into matrix as shown in (15), where $X$ is a matrix of mixture of source signals, represented by the voltage receiver $\left(V_{\mathrm{R}}\right) \cdot \exp (-\alpha l)$ is denoted as a mixing matrix $(A)$ and voltage transmitter $\left(V_{\mathrm{T}}\right)$ is

The equations for receiver RX2 voltage, receiver RX3 voltage,

$$
\begin{aligned}
V_{\mathrm{R} 2}= & V_{\mathrm{T} 1} \exp \left(-\alpha_{2} l_{2}-\alpha_{3} l_{3}\right)+V_{\mathrm{T} 2} \exp \left(-\alpha_{3} l_{1}\right) \\
& +V_{\mathrm{T} 3} \exp \left(-\alpha_{3} l_{1}\right)+V_{\mathrm{T} 4} \exp \left(-\alpha_{4} l_{2}-\alpha_{3} l_{3}\right), \\
V_{\mathrm{R} 3}= & V_{\mathrm{T} 1} \exp \left(-\alpha_{1} l_{2}-\alpha_{4} l_{3}\right)+V_{\mathrm{T} 2} \exp \left(-\alpha_{3} l_{2}-\alpha_{4} l_{3}\right) \\
& +V_{\mathrm{T} 3} \exp \left(-\alpha_{4} l_{1}\right)+V_{\mathrm{T} 4} \exp \left(-\alpha_{4} l_{1}\right), \\
V_{\mathrm{R} 4}= & V_{\mathrm{T} 1} \exp \left(-\alpha_{1} l_{1}\right)+V_{\mathrm{T} 2} \exp \left(-\alpha_{2} l_{2}-\alpha_{1} l_{3}\right) \\
& +V_{\mathrm{T} 3} \exp \left(-\alpha_{4} l_{2}-\alpha_{1} l_{3}\right)+V_{\mathrm{T} 4} \exp \left(-\alpha_{1} l_{1}\right) .
\end{aligned}
$$

ICA is utilized to separate the mixture of source signals where in (16) the matrix positioned of source signals $(S)$ represented as $V_{\mathrm{T}}$ is interchanged with $V_{\mathrm{R}}$ denoted by matrix of mixture of source signals $(X)$. The separating matrix $(W)$ is formed to replace the mixing matrix $(A)$ where theoretically $W$ is obtained by the inversing process of $A$ :

$$
\begin{gathered}
\widehat{S}= \\
{\left[\begin{array}{c}
V_{\mathrm{T} 1} \\
V_{\mathrm{T} 2} \\
V_{\mathrm{T} 3} \\
V_{\mathrm{T} 4}
\end{array}\right]=\left[\begin{array}{llll}
W_{1,1} & W_{1,2} & W_{1,3} & W_{1,4} \\
W_{2,1} & W_{2,2} & W_{2,3} & W_{2,4} \\
W_{3,1} & W_{3,2} & W_{3,3} & W_{3,4} \\
W_{4,1} & W_{4,2} & W_{4,3} & W_{4,4}
\end{array}\right]\left[\begin{array}{c}
V_{\mathrm{R} 1} \\
V_{\mathrm{R} 2} \\
V_{\mathrm{R} 3} \\
V_{\mathrm{R} 4}
\end{array}\right] .}
\end{gathered}
$$

In this case, the parameter considered for computing the concentration profile of bubble flow is the matrix $A$. However, the matrix cannot be acquired directly from the ICA method. Hence, the related parameter regarding $A$ is supported where the matrix $W$ is obtained as an inverse process in order to find the matrix $A$.

\section{Hardware Design}

In the optical tomography system, eighteen transmitters and eighteen receivers are mounted around a 100-millimeter ( $\mathrm{mm})$ diameter pipe by using a sensor jig. The transparent pipe is made from an acrylic material which allows visual observation of the flow process. The transmitters are positioned next to the receivers since the system is modelled based on fan beam light projection. The infrared Light-Emitting Diode
(LED) model TSAL6200 is used as the transmitters where it emits an invisible light. The invisible light has a smaller value of wavelength compared to the visible light in which the range is 750 nanometers $(\mathrm{nm})$ and above. The photodiode model BPV10NF is selected due to its ability to detect the infrared light from the transmitter. A frequency of $50 \mathrm{Hertz}$ $(\mathrm{Hz})$ is chosen for the transmitting circuit frequency, and each transmitter is set using different pulse durations for switching time by using a Peripheral Interface Controller (PIC) Microcontroller. Transmitter 1 (TX1) is set to 1 millisecond (ms) and TX2 for $2 \mathrm{~ms}$ and followed until $18 \mathrm{~ms}$ for TX18. The purpose for doing this is to facilitate the determination of the transmitter's signal detected by the photodiode where it will be explained in Section 4. The outputs from the receivers are connected to a signal conditioning circuit in order to convert the receiving light into voltage and for voltage amplification. The amplified voltage signals are converted into the digital form using the data acquisition system (DAQ) model U2331 manufactured by Agilent Technologies Inc. It has 3 megasamples per second (MS/s) of sampling rate, 64 single ended/32 differential inputs for analog input channels, and 24-bit programmable TTL input/output channels. The experiment is performed offline. The voltage output from receiver is converted into a digital form using the DAQ and further analysis is performed using the LabVIEW software, which provided the graphical based system for the ICA algorithm. The concentration profiles of the bubble's condition are displayed on a computer. The total time to obtain the final result of concentration profile is around 1 minute. An illustration of the overall design of the optical tomography system is shown in Figure 7. 


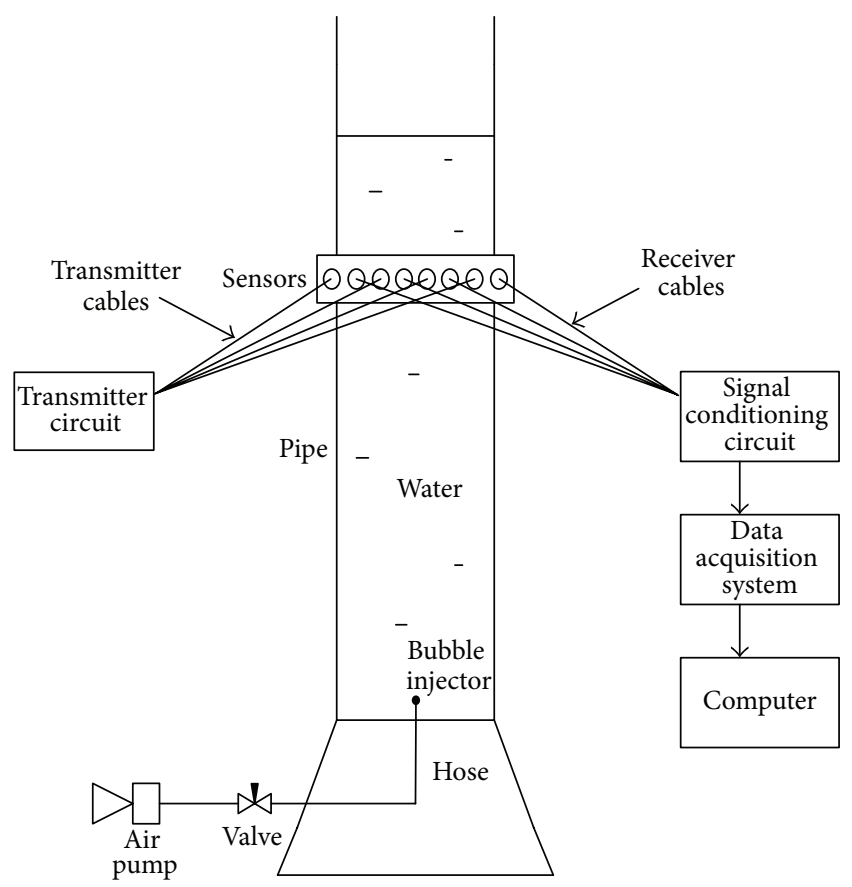

(a)

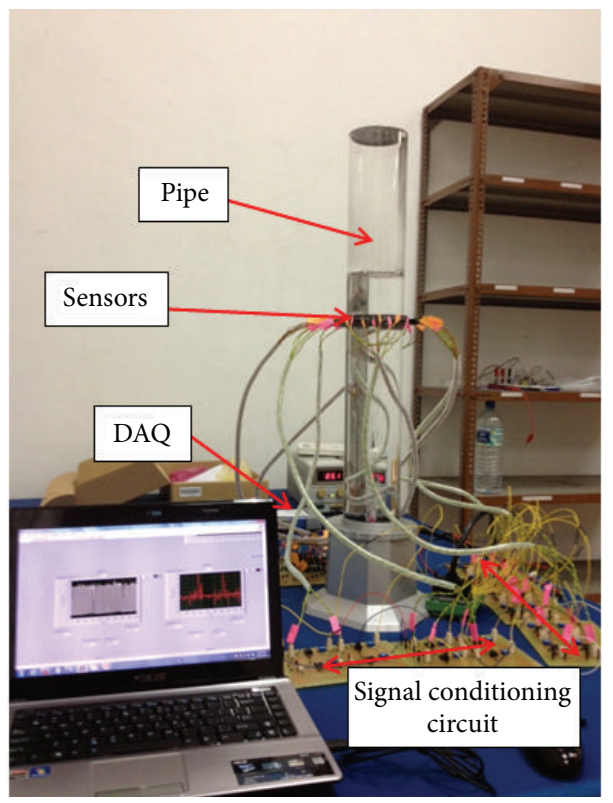

(b)

FIgURE 7: (a) The overall design of the optical tomography system. (b) The preparation of the apparatus.

\section{Experimental Procedure}

This section will elaborate the approach to calculate and analyse the result of bubble flow using ICA method. The steps of the experiment are according to these stages:

(1) Each transmitter has a unique value where it is programmed with different pulse duration value. Hence, the switching times are different from each other. It is assumed that there are eighteen signals from transmitters to be detected by each receiving sensor. Then, the ICA method is applied in order to separate the eighteen signals in order to get a single transmitter signal in each output indicator. The pulse duration value for the transmitter can be from $1 \mathrm{~ms}$ to $18 \mathrm{~ms}$, which is set in the transmitter circuit.

(2) A transmitter's signal at each output indicator also brings along a row of unmixed matrix $(W)$. The whole 18 rows for $W$ are produced in arbitrary rows and rearrangement of the matrix's row is necessary according to the order of transmitters pulse duration. For example, a signal with $10 \mathrm{~ms}$ is shown in output indicator; hence the value at row 1 will be shifted to row 10 . In other words, the rearrangement process for the row depends on the value of pulse duration displayed at each output indicator.

(3) The new matrix $W$ is formed using the ICA method. The matrix of $W$ is emphasized in the steps since it contains an important parameter that has a relationship with the mixing matrix $(A)$. In the ICA theory, $W=A^{-1}$. Hence, the $W$ matrix will be inversed to get the value of $A$ which consists of matrix $18 \times 18$ and expansion of $\exp (-\alpha l)$. The matrix of $A$ is named as the turbidity factor $(M)$.

(4) The 18 columns and 18 rows contained in matrix $A$ represented 18 receivers and 18 transmitters, respectively. Each value is represented as $M_{e, f}$ where $e$ is the transmitter number and $f$ is the receiver number. For example, by referring to Table $1, M_{2,1}$ has the value 2.782 . It indicates that when light is transmitted from transmitter 2 (TX2) to receiver 1 (RX1), it has a turbidity factor $(M)$ of 2.782 .

(5) The value at each pixel $(P)$ contained in the cross section of the pipe has to be determined. The $P$ value is the affiliation between $M$ and light's path length, where light crossed a pixel. An example of the light path along the pixels can be seen in Figure 8. For light transmitted from transmitter TX2 to receiver RX1, two pixels $\{1,11\}$ and $\{1,12\}$ are traversed by the light. The light's lengths in both pixels are measured as $0.4 \mathrm{~cm}$ and $0.5 \mathrm{~cm}$, respectively, with the total light's length as $0.9 \mathrm{~cm}$. The $P$ value is computed in order to know the quantity of light that has crossed pixels $\{1,11\}$ and $\{1,12\}$. The calculation started from pixel $\{1,6\}$, followed by other pixels contained in the pipe's cross section by using

$$
P_{r, c}=\frac{u}{t} \times M_{e, f},
$$

where $P_{r, c}$ is the pixel at row $(r)$ and column (c), $u$ is the path length of light that has crossed the pixel in centimeter $(\mathrm{cm}), t$ is the total length from 


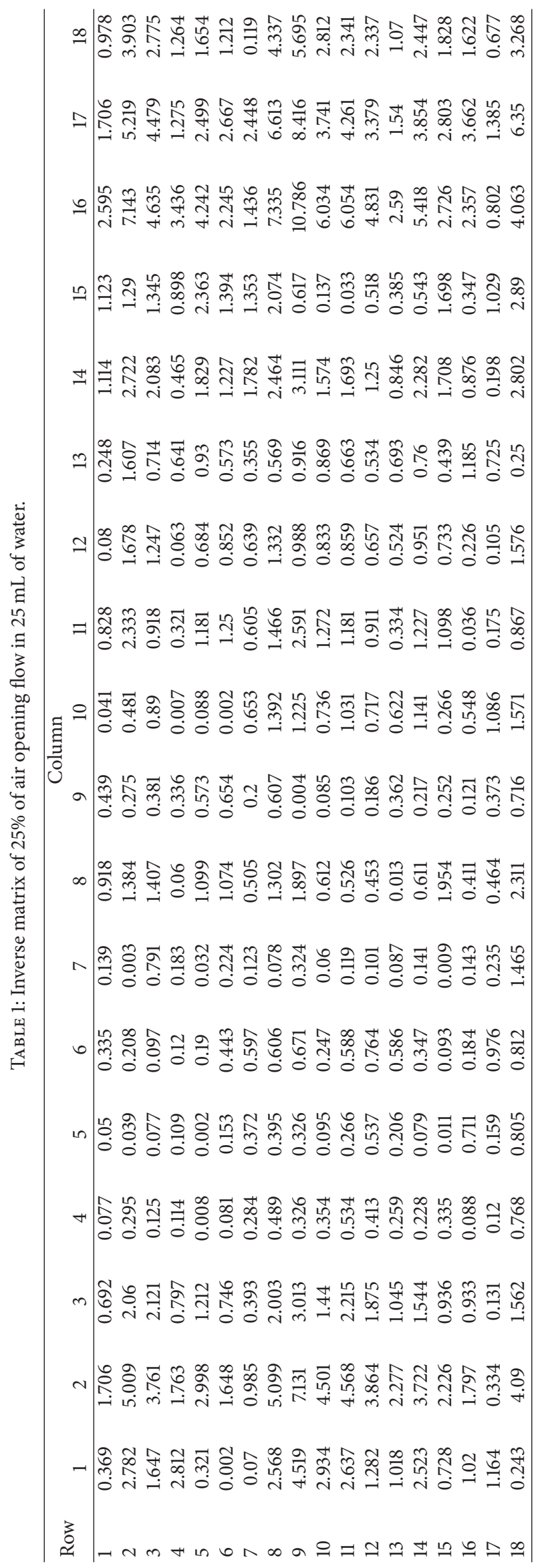




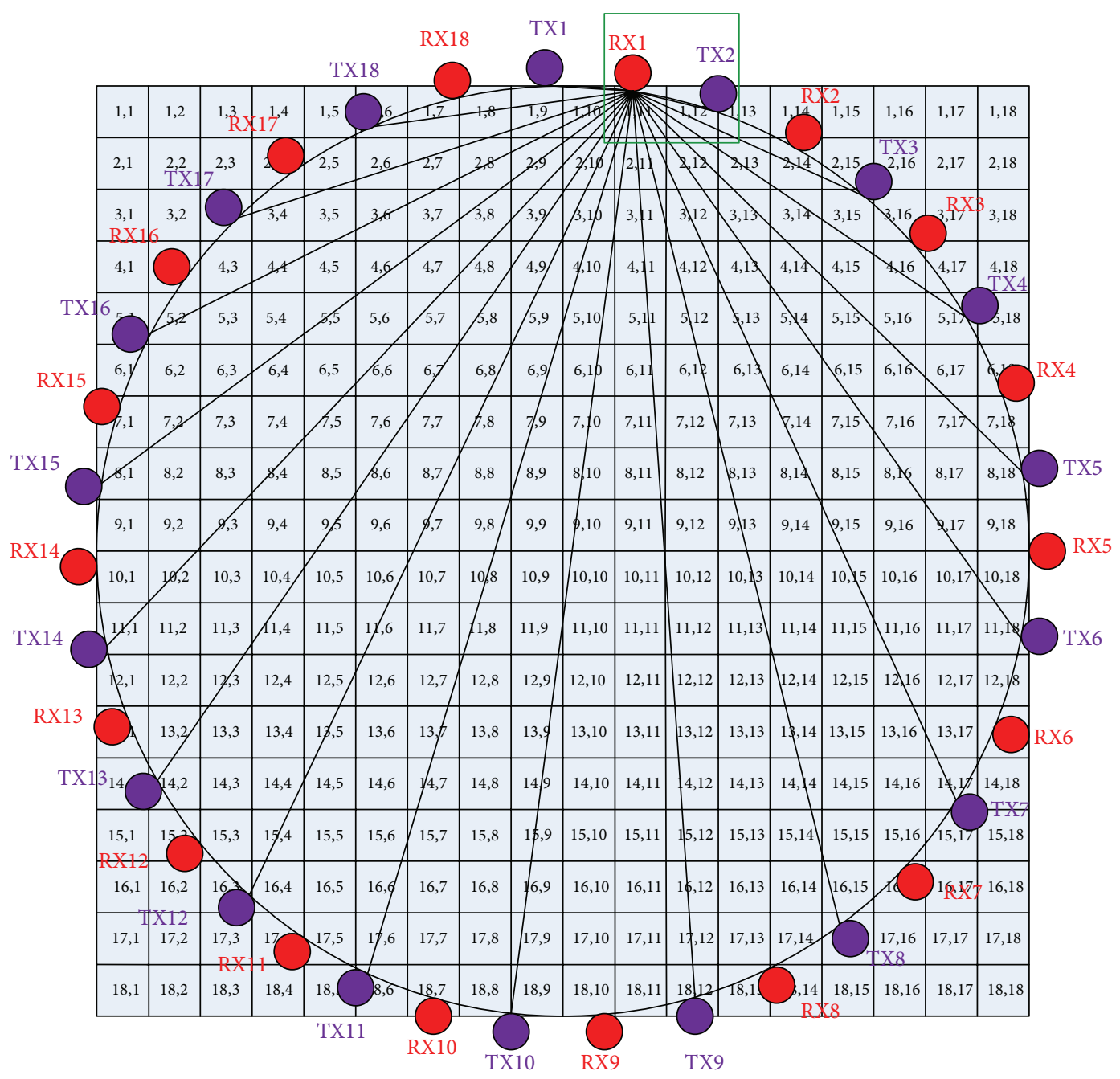

FIGURE 8: The analysis of transmitting light to receiver RX1.

the transmitter to the receiver, and $M_{e, f}$ is the turbidity factor. An example for calculating the $P$ value for pixel $\{1,12\}$ can be shown as

$$
\begin{aligned}
& P_{1,12}=\frac{0.5}{0.9} \times 2.782, \\
& P_{1,12}=1.546
\end{aligned}
$$

(6) From Figure 8, pixel $\{1,12\}$ is intercepted by four lights, which are from transmitters TX2, TX3, TX4, and TX5. By referring to Table 1, the $M$ value for light from transmitter TX3 to receiver RX1 is $M_{3,1}=1.647$. Then, the $P$ value is recalculated using (18) based on its light's path length. The calculation process for getting the $P$ value continued to other light sources, namely, transmitter TX4 and transmitter TX5.

(7) The transmitter's light also could cross pixel $\{1,12\}$ when it is transmitted to receiver RX2 until receiver $\mathrm{RX} 18$. Hence, it is necessary to recognise whether light from the transmitters has crossed the pixel or not. If it has, the $P$ values from all transmitters are totalled up and divide into the number of lights that has passed through pixel $\{1,12\}$. The result from this calculation is named as the average value $(K)$. The $K$ value is the final value to be computed in order to estimate the location of bubbles at certain pixels. Detail of the calculation is shown in Table 2.

\section{Result and Discussion}

This section presents the concentration profiles of gas bubbles flow in the vertical pipe filled with contaminated water. Five types of bubble flowing conditions are selected which are a single bubble flow, double bubble flow, and 25\%, 50\%, and $100 \%$ of air opening flow. Table 3 shows the gas bubble condition and concentration profile for a single bubble and double bubbles flow in contaminated water. The sample of contaminated water is prepared by adding $25 \mathrm{~mL}$ of green 


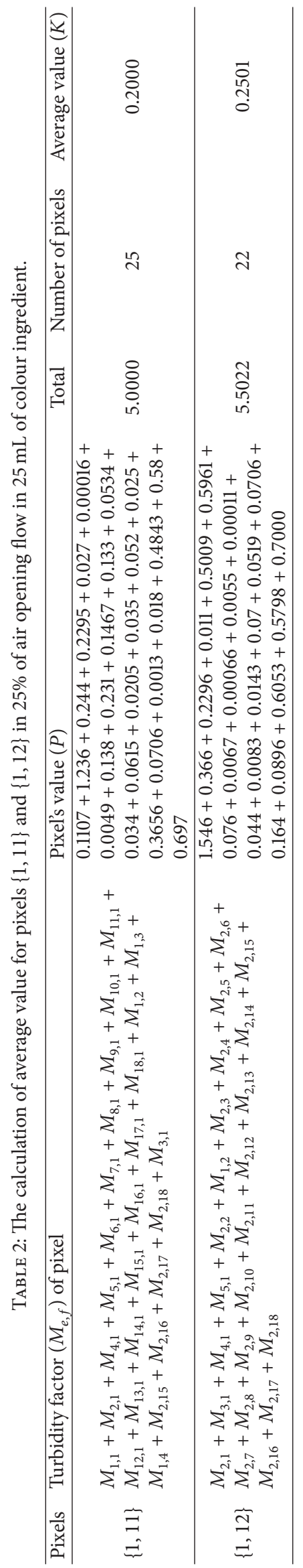


TABLE 3: The gas bubble condition and concentration profile for a single bubble and double bubble in contaminated water.

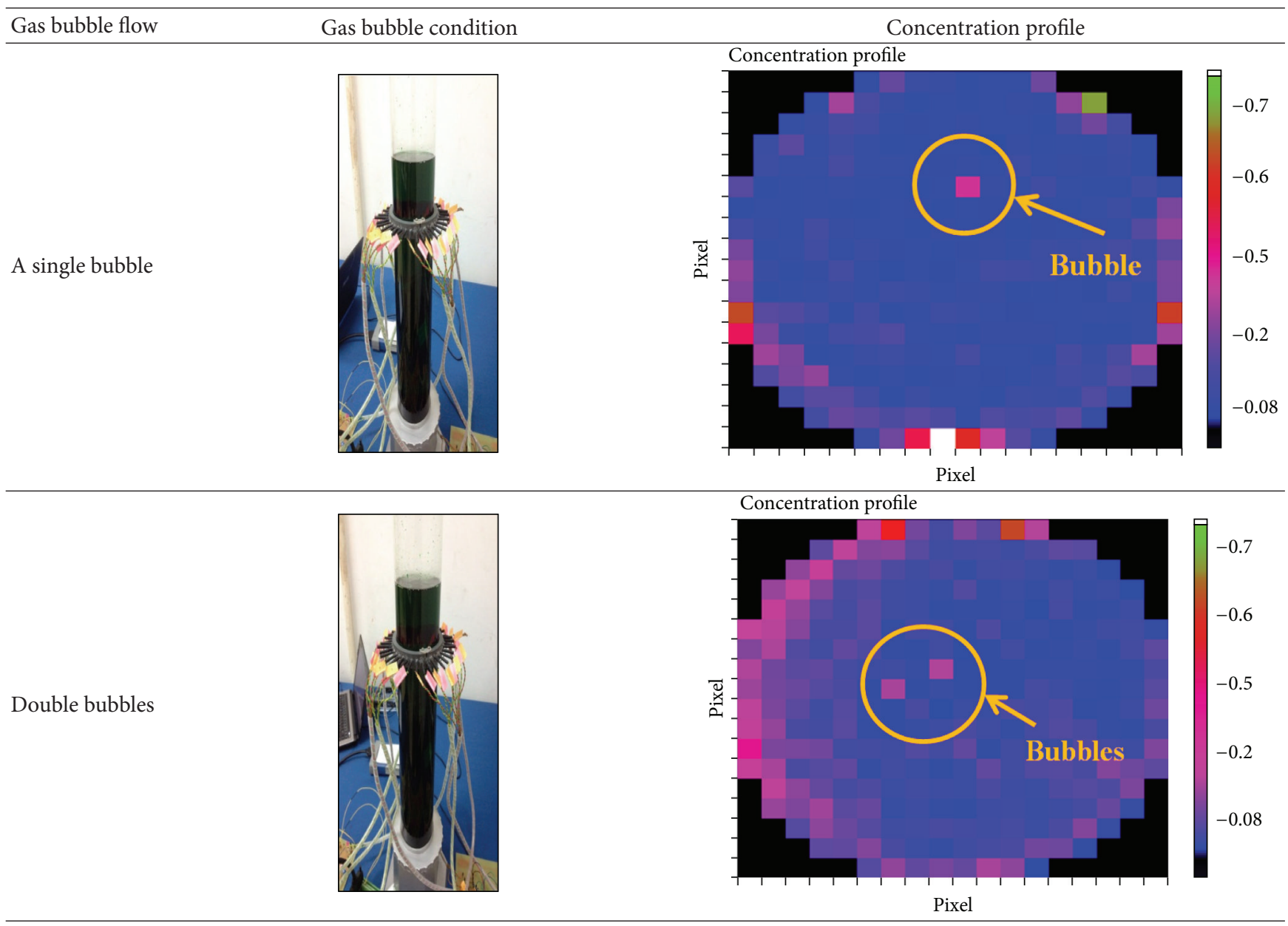

food colouring to the pipe which contains 3 liters of pure water filled in the pipe. The limitation of this research is that the experiment cannot be performed in volume higher than $25 \mathrm{~mL}$ such as $30 \mathrm{~mL}$ or $35 \mathrm{~mL}$ of green contaminated water. This is due to the fact that the ICA cannot separate the source signals properly since the water sample is too dark.

5.1. 25\% of Air Opening. The experiments were conducted by opening up a quarter of the valve to allow the $25 \%$ volume of gas entering upwards in the vertical pipe as shown in Figure 9. The transmitter circuit is switched on, before the LabVIEW software is run to apply the ICA algorithm. The $K$ value is computed using the Matlab software by entering the inverse matrix $(A)$ acquired by the ICA process.

Table 4 shows the result of $K$ value for $25 \%$ of air opening flow. The location of bubbles is estimated by analysing the value of $K$. By referring to the mathematical model that has been discussed in considering the presence of gas bubbles, the value of receiver voltage $V_{\mathrm{R}}$ is getting high compared to $V_{\mathrm{R}}$ in homogeneous phase flow. The high value of $V_{\mathrm{R}}$ is related to $\exp (-\alpha l)$ which varied when gas bubbles exist. The properties of $\exp (-\alpha l)$ are the outcome for $A$ matrix and have influenced

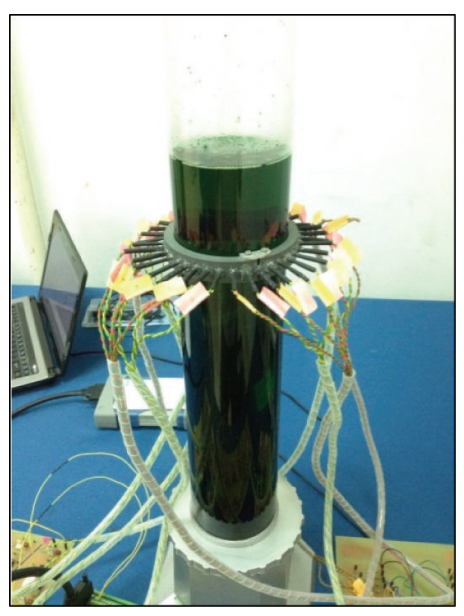

FIGURE 9: The condition of $25 \%$ of air opening.

the calculation of $K$ parameter. The range of values of $K$ is considered from 0.1 and above. Therefore, the bubbles are supposed to be located in the pixels which contain at least 0.1 . 


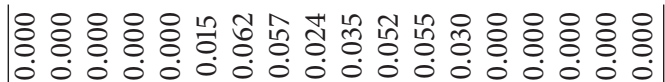

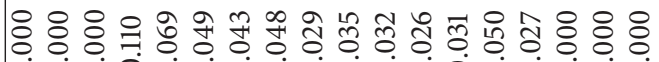

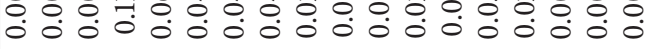

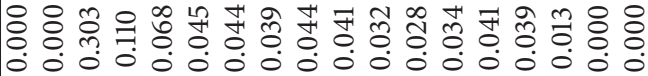

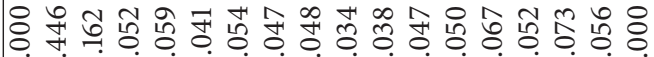

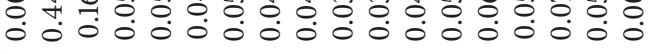

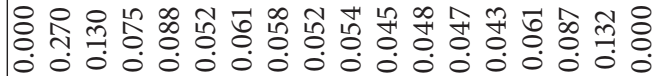

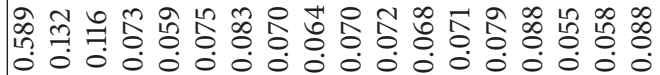

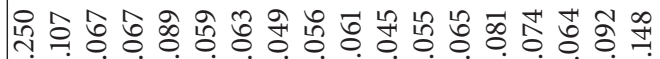
言

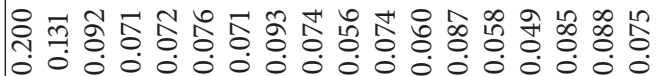

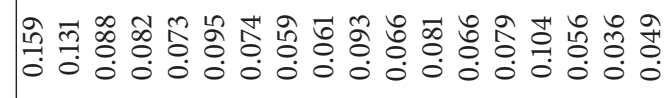
in

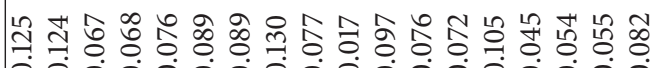

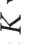

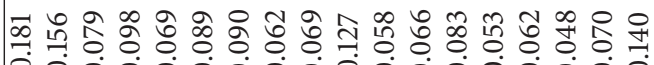
栗

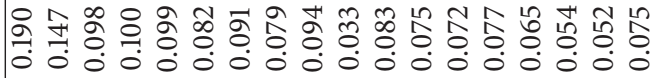

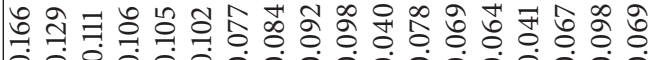

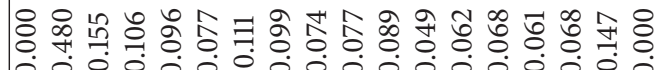

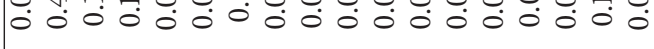

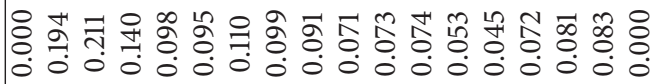

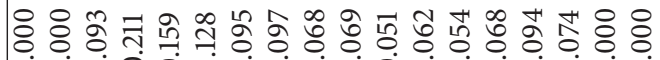
2.8ว

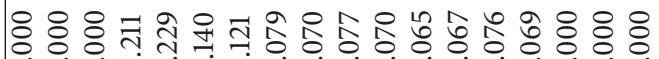

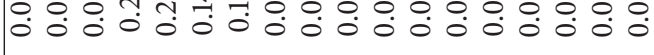

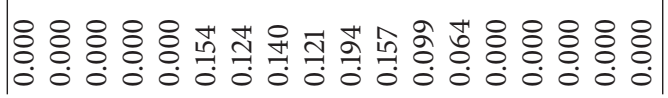




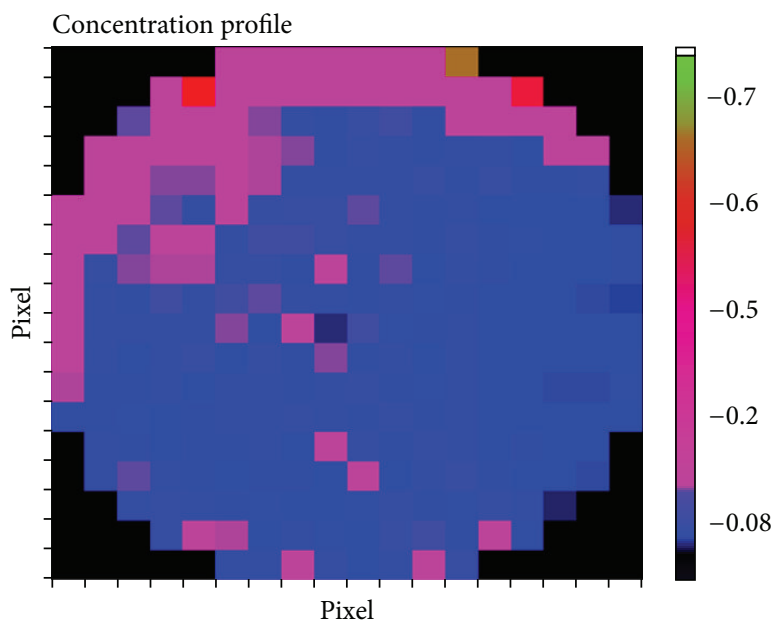

FIgURE 10: The concentration profile for $25 \%$ of air opening.

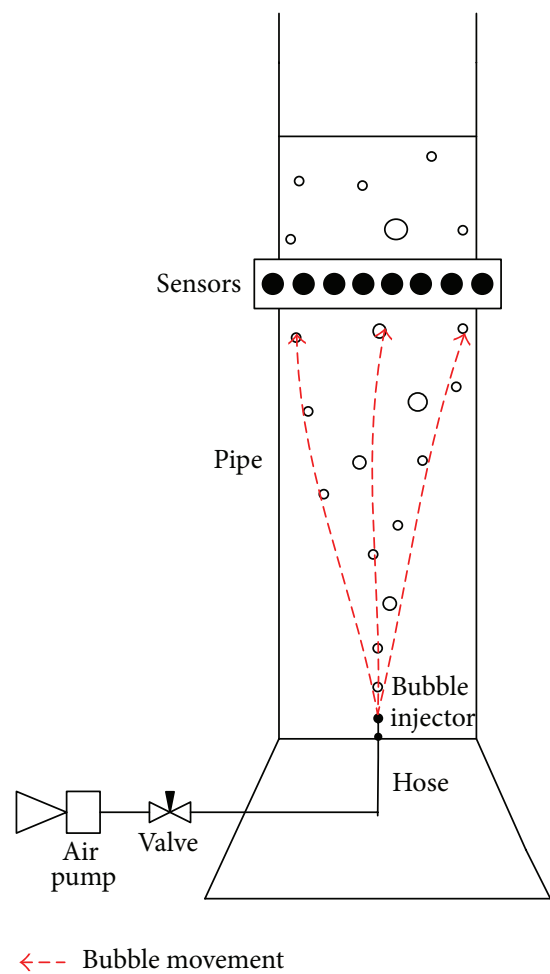

FIgURE 11: Bubbles movement upwards from the bottom.

The result in Table 4 is transformed to concentration profile as shown in Figure 10 in order to visualize bubble location. The pink colour in the concentration profile indicates that some bubbles are inclined to rise towards to the border of the pipe although the bubble injector is installed at the centre of the pipe base. The illustration for the bubble's movements can be seen in Figure 11. Other than that, the high value of pixels located near the pipe's periphery is probably due to the existence of receivers around the pipe's periphery. Hence, most of the light is likely to cross the pixels around the pipe's periphery. Although the average value is calculated, the $K$ value at the pipe's periphery is still higher compared to other locations.

5.2. 50\% of Air Opening. Half of the valve is opened in this experiment as in Figure 12(a) to ensure that more bubbles are inserted into the pipe than the first experiment. The concentration profile for the medium bubble flow is shown in Figure 12(b). The result of $K$ value is higher compared to the $25 \%$ of air opening where the range for bubble is from 0.12 to 0.5 . It is due to the light's energy experiencing more attenuation when the bubbles interrupted the light path as modelled in (4) to (6). Moreover, the bubbles also became 


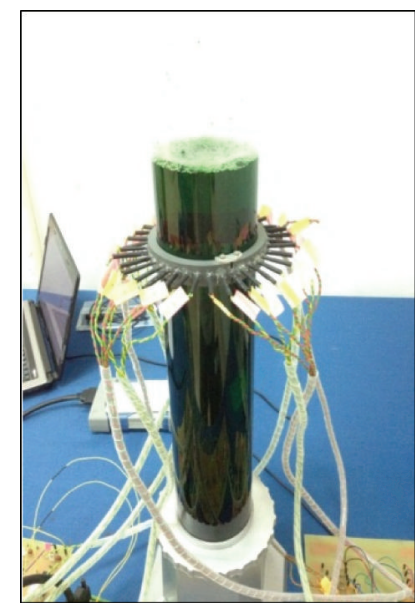

(a)

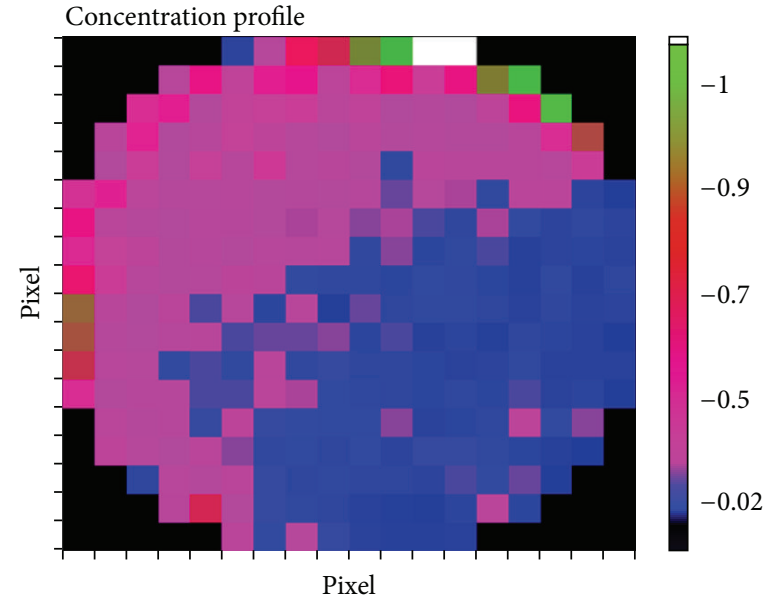

(b)

FIgURE 12: (a) The condition of 50\% of air opening. (b) The concentration profile for $50 \%$ of air opening.

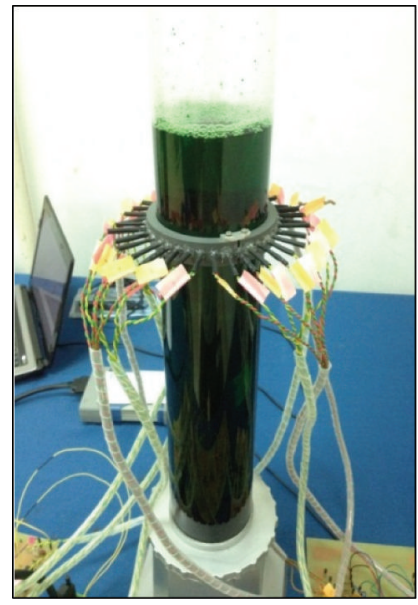

(a)

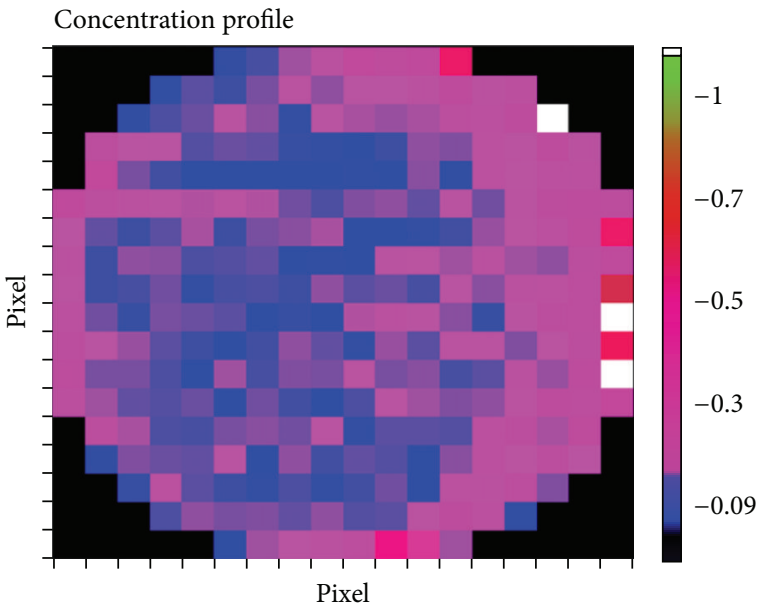

(b)

FIgURE 13: (a) The condition of $100 \%$ of air opening. (b) The concentration profile for $100 \%$ of air opening.

close to each other when more bubbles went upward since the space around the pipe is limited and compact. Thus, the collision between bubbles occurred and generated the additional energy which resulted in the value of $\exp (-\alpha l)$ increasing.

5.3. 100\% of Air Opening. In this experiment, the valve is fully open and the bubble flow condition is shown in Figure 13(a). The range of bubble is from 0.2 to 0.5 , and this represents an increase compared to medium bubble flow. The location of bubble can be seen in Figure 13(b) which shows that most of the area in the cross section of the pipe is occupied by gas bubbles. Thus, all sensors in the system can detect the presence of bubble in high bubble flow condition. This condition also creates more collision between the bubbles than medium bubble flow since the free space in the pipe has been reduced by the addition of gas bubbles. More collisions of bubbles have influenced the reading in terms of the increasing value of $K$.

\section{Conclusion}

In this paper, an optical tomography system using the ICA method has been presented to estimate the distribution of bubbles in a vertical pipeline. The system proved its ability to obtain information on the gas bubbles concentration profile in the pipe without disturbing the process flow. The concentration profiles for the bubble flow quantities are investigated under five different flows conditions: a single bubble, double bubbles, $25 \%$ of air opening, $50 \%$ of air opening, and $100 \%$ of air opening flow rates. The ICA method has a great potential in improving the process efficiency by visualizing the concentration profiles inside the pipelines.

\section{Appendix}

See Tables 5-9. 


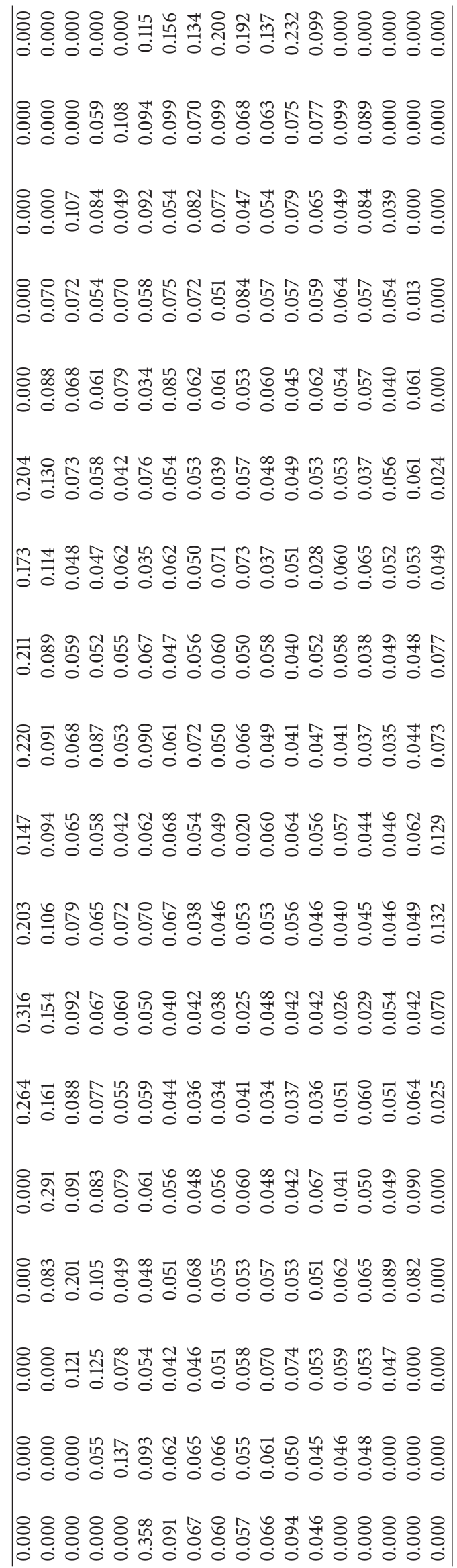




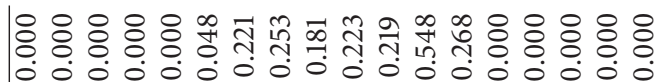

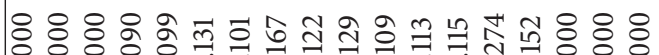

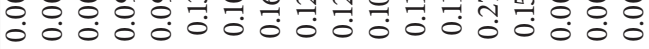

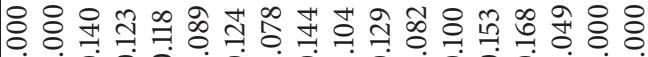

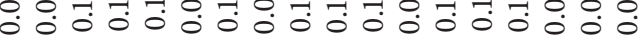

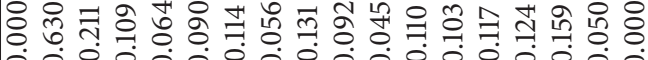

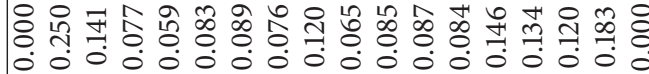

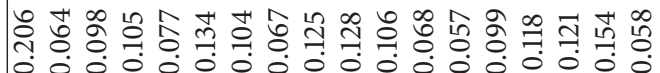

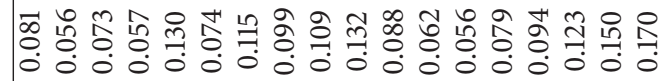

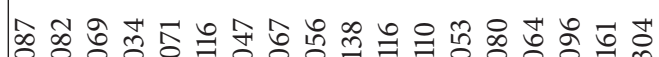

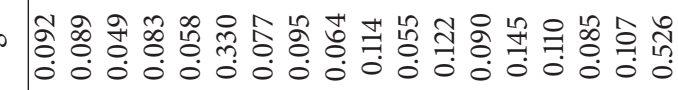
त्ञ

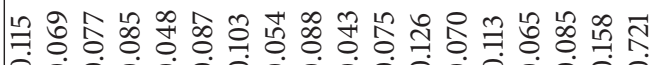
$\ddot{6}$

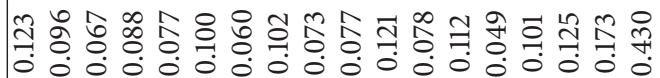

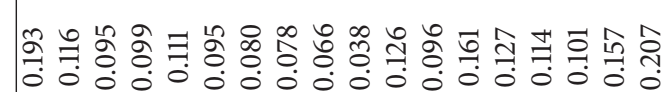

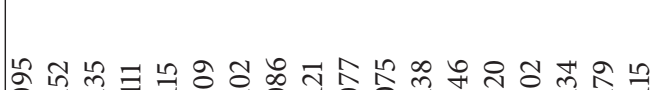

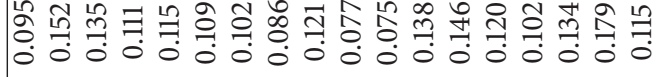

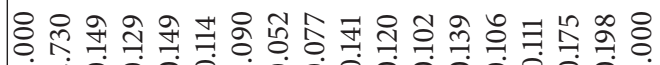

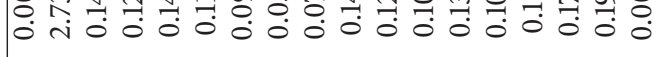

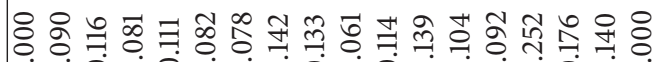

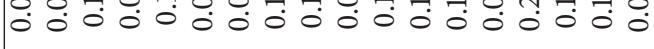

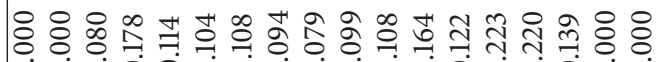

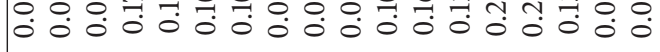

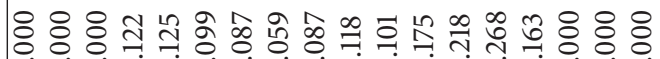

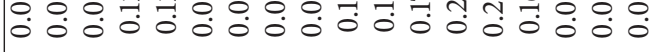

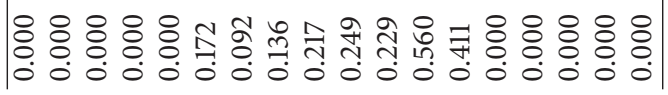




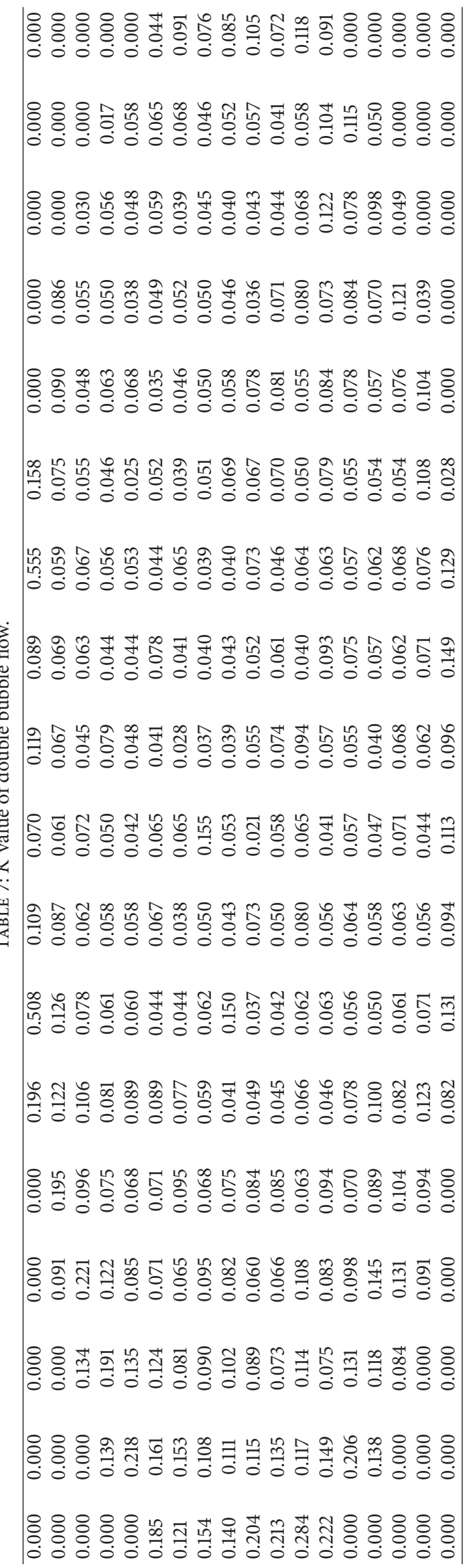




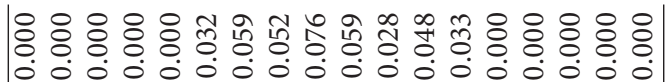

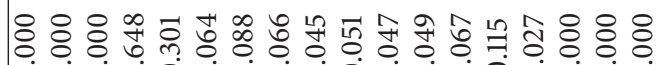

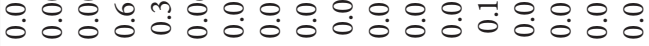

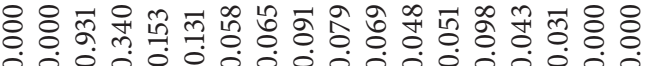

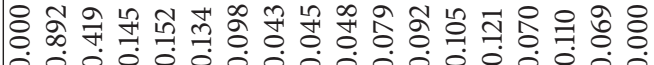

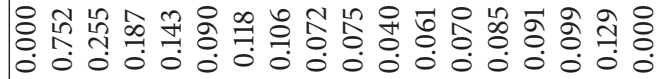

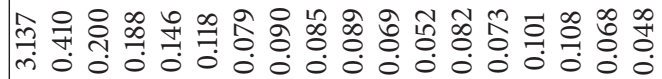

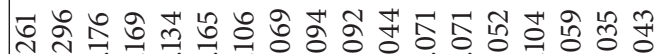
言

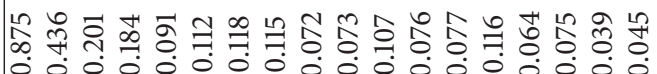

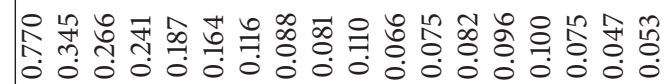
in

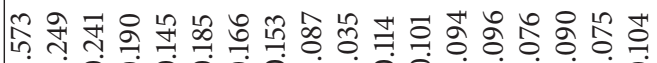
1

死全 范

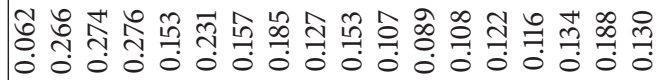

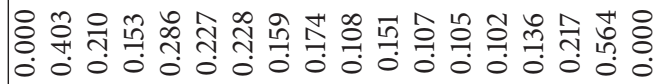

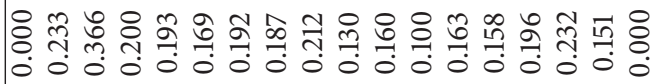

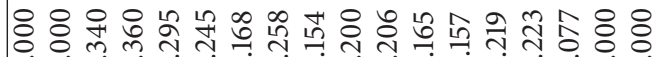
잉 흥응응열옹

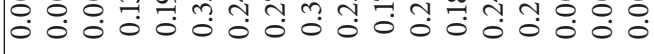

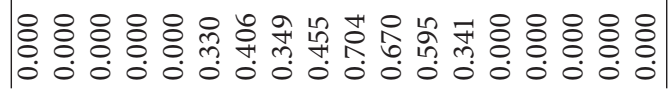




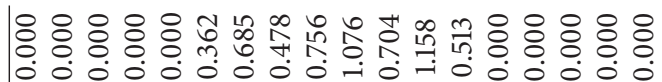

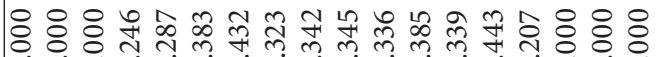

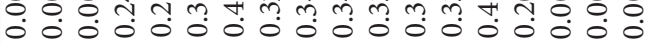

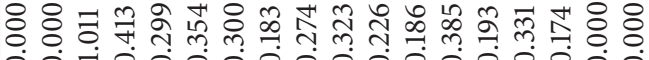

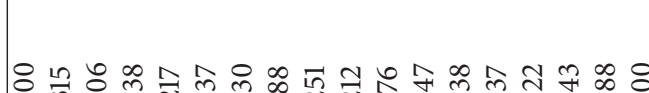

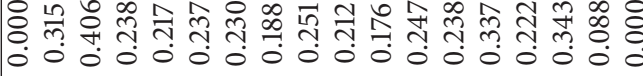
응

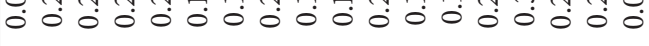

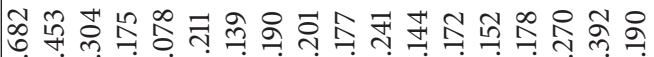

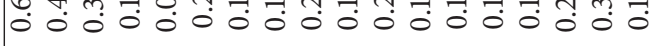

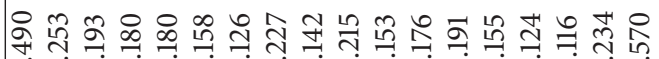
高

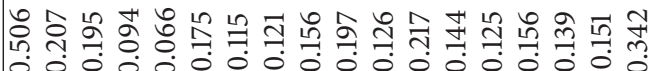

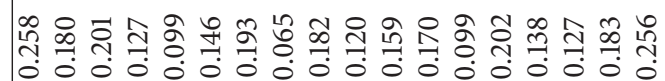

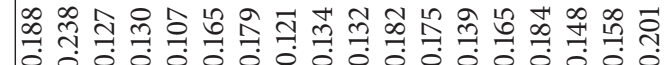
运

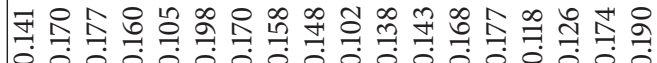

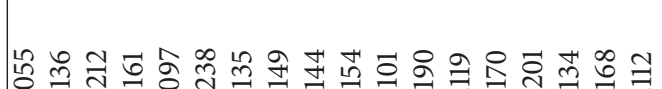

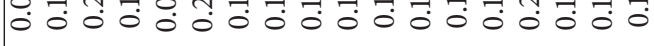

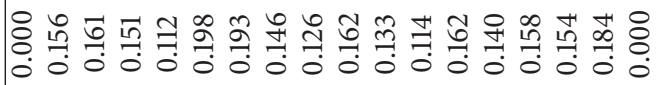

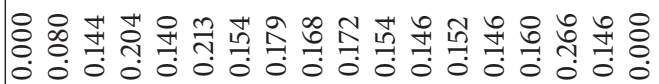

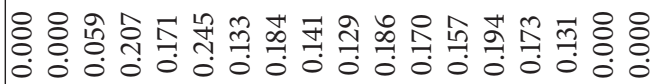

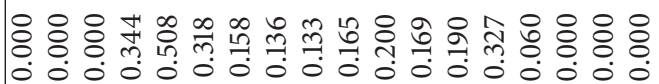

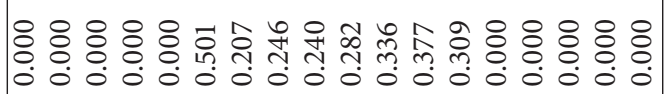




\section{Competing Interests}

The authors declare that there are no competing interests regarding the publication of this paper.

\section{Acknowledgments}

The authors wish to acknowledge the assistance of the Ministry of Higher Education, Malaysia, and Universiti Teknologi Malaysia under the GUP Research Vote $05 \mathrm{H} 67$ for providing the funds and resources in carrying out this research.

\section{References}

[1] C. Yan, J. Zhong, Y. Liao, S. Lai, M. Zhang, and D. Gao, "Design of an applied optical fiber process tomography system," Sensors and Actuators B: Chemical, vol. 104, no. 2, pp. 324-331, 2005.

[2] D. L. George, J. R. Torczynski, K. A. Shollenberger, and T. J. O'Hern, "Validation of electrical-impedance tomography for measurements of material distribution in two-phase flows," International Journal of Multiphase Flow, vol. 26, no. 4, pp. 549$581,2000$.

[3] T. Dyakowski, L. F. C. Jeanmeure, and A. J. Jaworski, "Applications of electrical tomography for gas-solids and liquid-solids flows-a review," Powder Technology, vol. 112, no. 3, pp. 174-192, 2000.

[4] R. Abdul Rahim, C. K. Thiam, and M. H. F. Rahiman, "An optical tomography system using a digital signal processor," Sensors, vol. 8, no. 4, pp. 2082-2103, 2008.

[5] M. Idroas, R. Abdul Rahim, M. H. Fazalul Rahiman, M. N. Ibrahim, and R. G. Green, "Design and development of a CCD based optical tomography measuring system for particle sizing identification," Measurement, vol. 44, no. 6, pp. 1096-1107, 2011.

[6] M. R. Rzasa and A. Plaskowski, "Application of optical tomography for measurements of aeration parameters in large water tanks," Measurement Science and Technology, vol. 14, no. 2, pp. 199-204, 2003.

[7] S. Ibrahim, M. A. M. Yunus, R. G. Green, and K. Dutton, "Concentration measurements of bubbles in a water column using an optical tomography system," ISA Transactions, vol. 51, no. 6, pp. 821-826, 2012.

[8] R. M. Detsch and R. N. Sharma, "The critical angle for gas bubble entrainment by plunging liquid jets," The Chemical Engineering Journal, vol. 44, no. 3, pp. 157-166, 1990.

[9] M. R. Rzasa, "The measuring method for tests of horizontal twophase gas-liquid flows, using optical and capacitance tomography," Nuclear Engineering and Design, vol. 239, no. 4, pp. 699707, 2009.

[10] H. Jin, Y. Lian, S. Yang, G. He, and Z. Guo, “The parameters measurement of air-water two phase flow using the electrical resistance tomography (ERT) technique in a bubble column," Flow Measurement and Instrumentation, vol. 31, pp. 55-60, 2013.

[11] N. M. N. Ayob, M. H. F. Rahiman, Z. Zakaria, S. Yaacob, and R. A. Rahim, "Detection of small gas bubble using ultrasonic transmission-mode tomography system," in Proceedings of the IEEE Symposium on Industrial Electronics \& Applications (ISIEA '11), pp. 165-170, IEEE, Penang, Malaysia, October 2010.

[12] A. R. Daniels, Dual modality tomography for the monitoring of constituent volumes in multi-component flows [Ph.D. thesis], Sheffield Hallam University, 1996.
[13] G. R. Naik and D. K. Kumar, "An overview of independent component analysis and its applications," Informatica, vol. 35, pp. 63-81, 2011.

[14] Y. Xu, H. Wang, Z. Cui, F. Dong, and Y. Yan, "Separation of gasliquid two-phase flow through independent component analysis," IEEE Transactions on Instrumentation and Measurement, vol. 59, no. 5, pp. 1294-1302, 2010.

[15] C. Jutten and J. Herault, "Blind separation of sources, part I: an adaptive algorithm based on neuromimetic architecture," Signal Processing, vol. 24, no. 1, pp. 1-10, 1991.

[16] J. Chen and X. Z. Wang, "A new approach to near-infrared spectral data analysis using independent component analysis," Journal of Chemical Information and Computer Sciences, vol. 41, no. 4, pp. 992-1001, 2001.

[17] J. P. Huang and J. Mar, "Combined ICA and FCA schemes for a hierarchical network," Wireless Personal Communications, vol. 28, no. 1, pp. 35-58, 2004.

[18] S. Fiori, "Overview of independent component analysis technique with an application to synthetic aperture radar (SAR) imagery processing," Neural Networks, vol. 16, no. 3-4, pp. 453467, 2003.

[19] C. J. James and C. W. Hesse, "Independent component analysis for biomedical signals (topical review)," Physiological Measurement, vol. 26, no. 1, pp. R15-R39, 2005.

[20] G. Wang, Q. Ding, and Z. Hou, "Independent component analysis and its applications in signal processing for analytical chemistry," TrAC-Trends in Analytical Chemistry, vol. 27, no. 4, pp. 368-376, 2008.

[21] A. Hyvärinen, "Fast and robust fixed-point algorithms for independent component analysis," IEEE Transactions on Neural Networks, vol. 10, no. 3, pp. 626-634, 1999.

[22] A. Hyvärinen and E. Oja, "Independent component analysis: algorithms and applications," Neural Networks, vol. 13, no. 4-5, pp. 411-430, 2000.

[23] A. J. Bell and T. J. Sejnowski, "An information-maximization approach to blind separation and blind deconvolution," Neural Computation, vol. 7, no. 6, pp. 1129-1159, 1995.

[24] J. H. Garvey, Independent component analysis by entropy maximization (Infomax) [M.S. thesis], Naval Postgraduate School, Monterey, Calif, USA, 2007. 

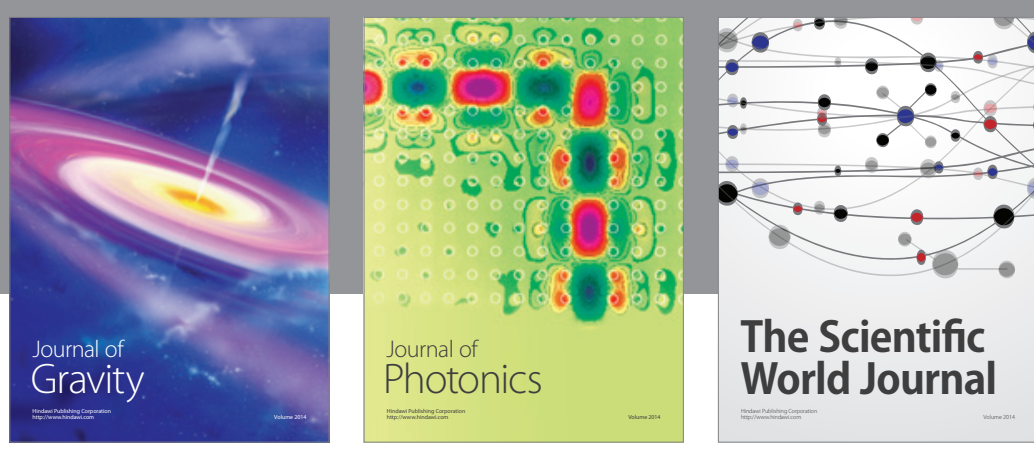

The Scientific World Journal
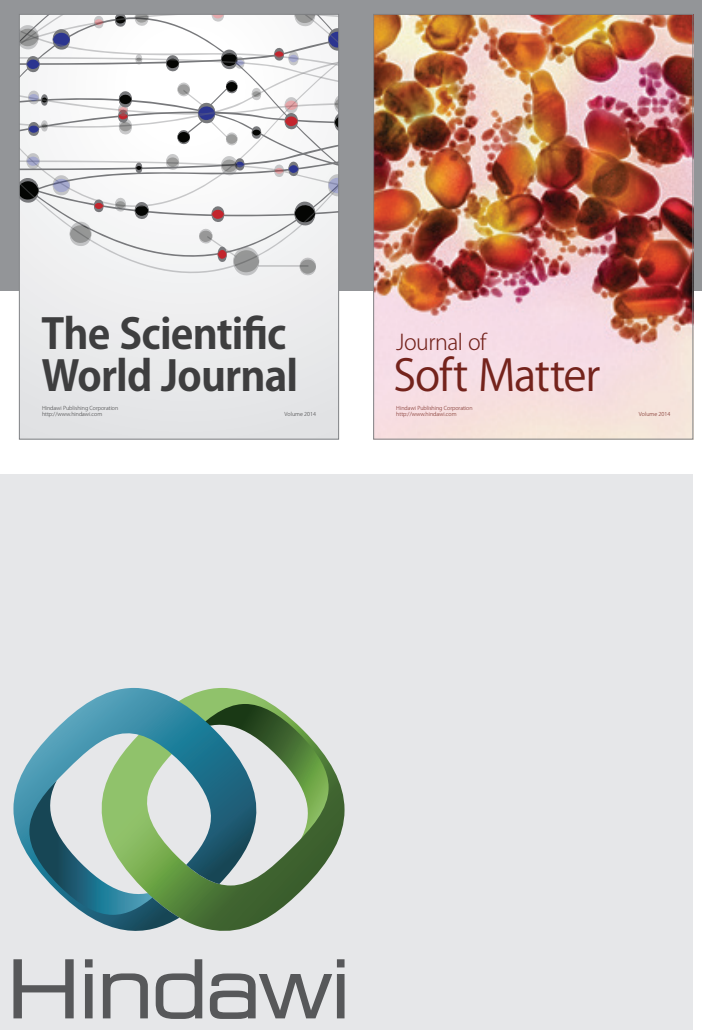

Submit your manuscripts at

http://www.hindawi.com

nternational Journal of

Statistical Mechanics
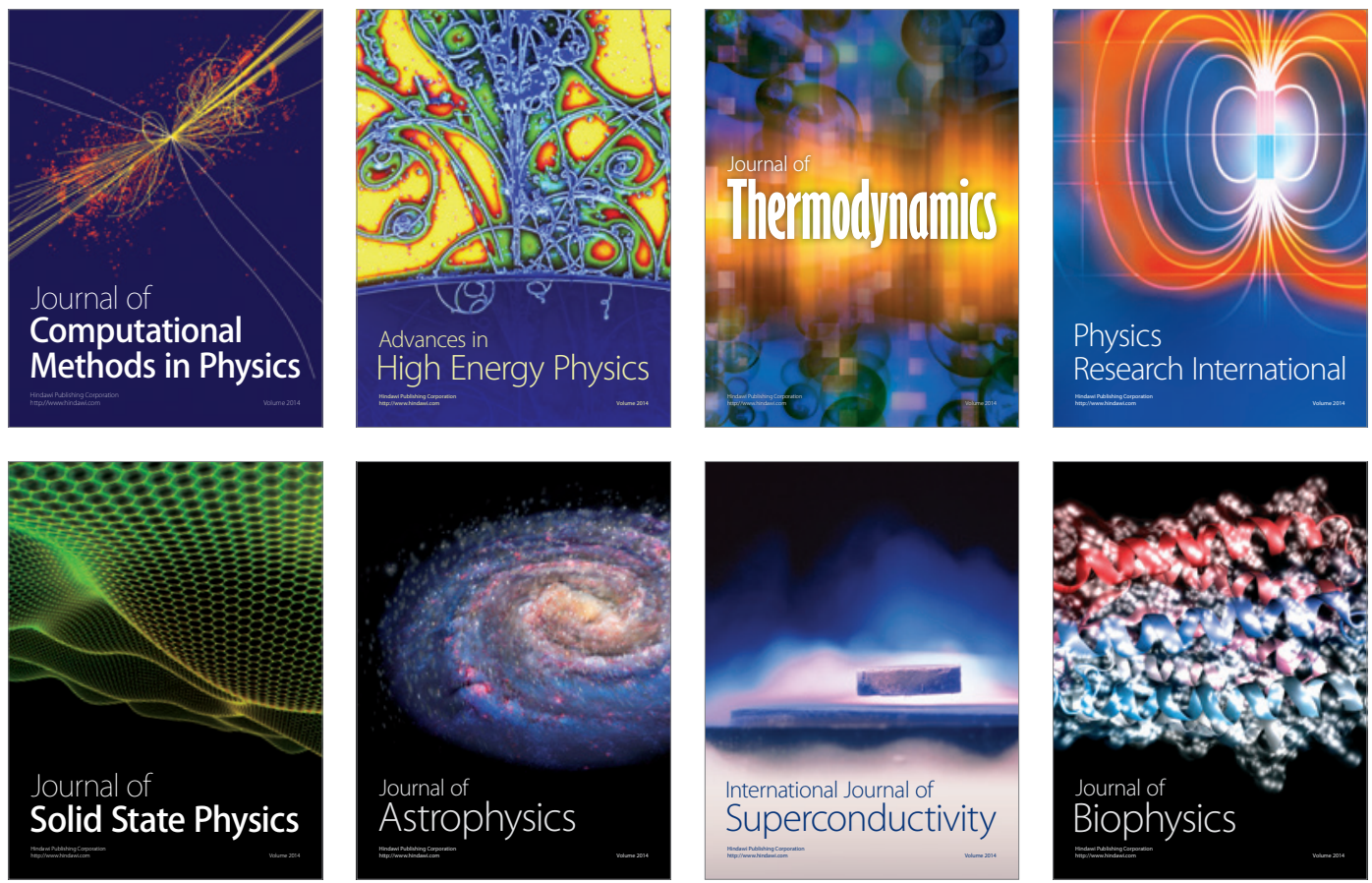
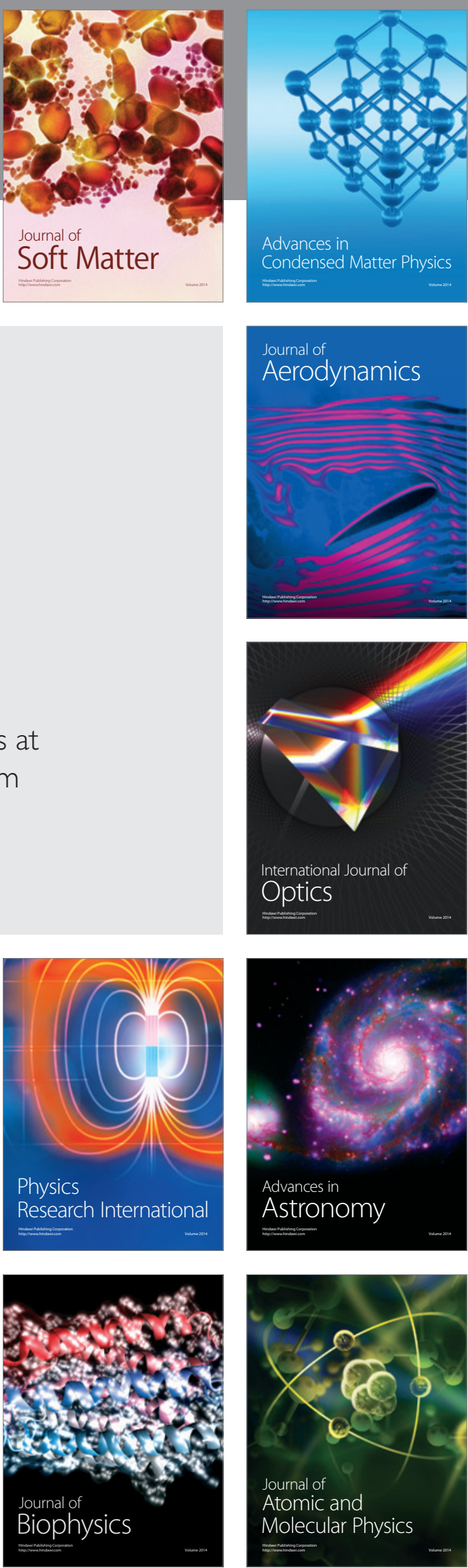